\title{
Parallel Transfer Optical Packet Switches
}

\author{
C. Y. Li, Member, IEEE, Ping kong Alexander Wai, Senior Member, IEEE, and Victor O. K. Li, Fellow, IEEE
}

\begin{abstract}
For efficient utilization of bandwidth in optical packet switching, the guard time $T_{\mathrm{g}}$ between packets should only be a small fraction of the packet transmission time $T_{\mathrm{d}}$. Since the guard time $T_{\mathrm{g}}$ of existing packet switching approaches must be larger than the reconfiguration time $T_{\mathrm{sw}}$ of optical switches, this imposes a stringent demand on the switch reconfiguration time $T_{\mathrm{sw}}$ as the transmission rate of optical fibers increases. By using batch transfer of packets or multiple switching fabrics in parallel, the requirement on the switch reconfiguration time can be significantly relaxed. The utilization of the transmission links can be greatly improved because the guard time between packets is no longer constrained by the switch reconfiguration time.
\end{abstract}

Index Terms-Blocking probability, optical switch, slotted optical network, switch reconfiguration time.

\section{INTRODUCTION}

$\mathbf{O}$ PTICAL network is one of the technologies that can provide the required transmission bandwidth for the rapidly growing communication traffic. Although terabits per second point-to-point transmission has been realized [1], a light path (wavelength channel) must be set up before any two nodes can exchange packets [2]. Owing to the lack of sophisticated optical signal processing devices and effective means to buffer light, all-optical packet switching is still in the research stage [3]. A more feasible approach of realizing optical packet switching is to optically switch the data packets but electronically process the packet headers for routing information. However, even such a hybrid approach is still difficult to realize.

One problem in implementing a practical optical packetswitched network is the difficulty of guaranteeing high-bandwidth utilization when the fiber transmission rate is high. In packet switched networks, a guard time $T_{\mathrm{g}}$ between packets is required to prevent packets from interfering with each other. In existing packet switches, packets are switched/transferred one by one. The packet guard time $T_{\mathrm{g}}$ must therefore be larger than the reconfiguration time $T_{\mathrm{sw}}$ of the switches. Otherwise, accidental packet discard may occur. Recently, very fast all-optical switching has been demonstrated. Thus, the packet exchange rate and link utilization should only be limited by the processing speed of the packet headers [4]. However, fast optical switches

Manuscript received July 08, 2008; revised November 05, 2008. First published April 17, 2009; current version published June 24, 2009. This work was supported by a grant from The Hong Kong Polytechnic University under Project 1-BBZB.

C. Y. Li and P. K. A. Wai are with the Photonics Research Center and Department of Electronic and Information Engineering, The Hong Kong Polytechnic University, Hung Hom, Hong Kong, China (e-mail: enli@ polyu.edu.hk; enwai@polyu.edu.hk).

V. O. K. Li is with the Department of Electrical and Electronic Engineering, The University of Hong Kong, Hong Kong, China (e-mail: vli@eee.hku.hk).

Color versions of one or more of the figures in this paper are available online at http://ieeexplore.ieee.org

Digital Object Identifier 10.1109/JLT.2008.2009549 with switching time $T_{\mathrm{sw}}$ in nanosecond or picosecond ranges are only available in small sizes such as $2 \times 2$ [4]. Large optical switches with up to a thousand ports have also been demonstrated using the microelectromechanical system (MEMS) technology but the required switch reconfiguration time $T_{\mathrm{sw}}$ is of the order of milliseconds [5]. Since no data transmission can occur during the guard time $T_{\mathrm{g}}$, a large guard time $T_{\mathrm{g}}$ will lead to low transmission bandwidth utilization. As the fiber transmission rate increases, the switch reconfiguration time $T_{\mathrm{sw}}$ will become increasingly significant in the determination of the transmission bandwidth utilization

In optical burst switching (OBS) networks [6], we have proposed to reduce the negative impact of large switch reconfiguration time $T_{\mathrm{sw}}$ by reconfiguring the optical switches before the arrival of packets [7]. This approach, however, requires retrieval of prior information of the switch status from the OBS reservation signaling, and may not be directly applicable to other types of optical packet-switched networks such as the slotted optical networks. Many important networks are slotted, for example the asynchronous transfer mode (ATM) networks [8] and the deflection routed networks [9]. The size of a time slot is in general a compromise between different considerations of traffic and network performance. Consequently, increasing the packet size to maintain reasonable throughput is not feasible.

Although slotted networks in general do not provide prior information of the switch status as in OBS networks, the features of fixed packet size and synchronous transmission allow other ways to tackle the problem such as by using batch transfer of packets or multiple switching fabrics working in parallel. Both methods can significantly relax the constraint imposed on the packet guard time $T_{\mathrm{g}}$ by the switching fabric reconfiguration time $T_{\mathrm{sw}}$. In batch transfer, a packet is not immediately routed to its desired output when the packet arrives at the input of the switch. The optical switch waits for $K$ packet transmission times before transferring the packets to their desired outputs. Since the packets arrive continuously, the optical switch may have to process at most $K$ packets in a batch. The required inputs and outputs of the switching fabric used in the proposed switch architecture should therefore be $K$ times that of the switches if batch transfer is not used. Since the number of connecting links between nodes (switches) in the network remains unchanged, 1-to- $K$ packet serial-to-parallel and $K$-to-1 packet parallel-to-serial converters are used. As the proposed optical packet switch transfers $K$ packets in a batch from inputs to outputs during $K$ packet transmission times, the time available for the switch reconfiguration is roughly $K-1$ packet transmission times.

Another way to relax the constraint on the packet guard time $T_{\mathrm{g}}$ is to use $K$ switching fabrics in parallel such that each switching fabric transfers only one packet during each $K$ 


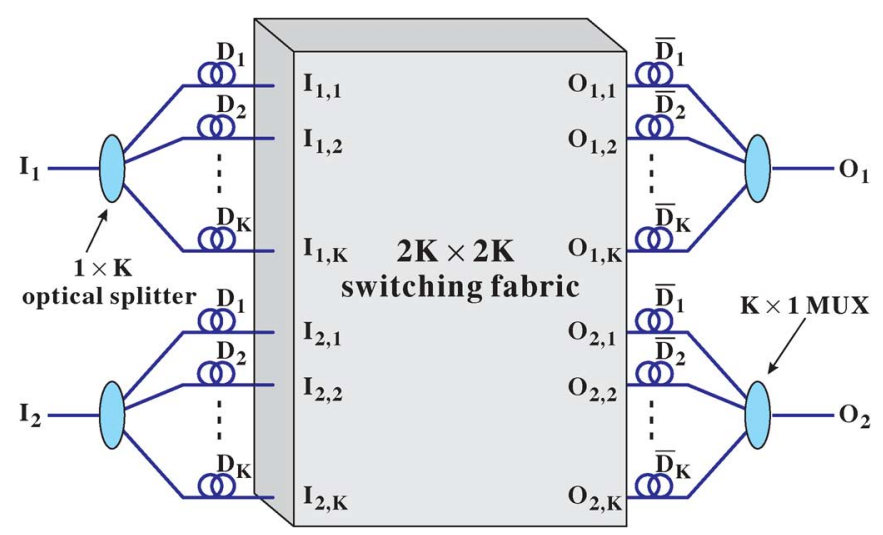

Fig. 1. MSBT switch architecture of a $2 \times 2$ optical switch. The $1 \times K$ optical splitter and its associated $K$ sets of FDLs form a packet serial-to-parallel converter. Also, the $K \times 1$ MUX and its associated $K$ sets of FDLs form the packet parallel-to-serial converter. The $2 K \times 2 K$ optical switch is assumed to be a commercially available internally nonblocking optical switch.

packet transmission time. Hence, each switching fabric will have $K-1$ packet transmission times to reconfigure itself. If the $K$ switching fabrics are scheduled properly, the guard time $T_{\mathrm{g}}$ between packets can also be smaller than the required time $T_{\mathrm{sw}}$ for a single switching fabric reconfiguration. In this paper, we propose and analyze the switch architectures that can be used to implement batch packet transfer or multiple switching fabrics in parallel in order to relax the requirement on the switching fabric reconfiguration time $T_{\mathrm{sw}}$. In Section II, we describe the proposed multislot batch-transfer (MSBT) switch architecture. The operation of packet serial-to-parallel transmission conversion and the timing for packet transfer are discussed. In MSBT, the added packet delay is large and requires large switching fabric. In Section III, we discuss the multifabric sequential transfer (MFST) architecture which assumes multiple switching fabrics in parallel. To further reduce the added packet delay in the MFST switch, we decouple the routing information from the packets. We describe MFST with pilot message (MFST/PM) switch architecture in Section IV. Section $\mathrm{V}$ gives the performance evaluation of the proposed switches including the link utilization, added packet delay, packet loss performance, and delay variance. We briefly discuss the implementation consideration in Section VI. Finally, we conclude the paper in Section VII.

\section{Multislot BATCH-TranSFER Switch}

Fig. 1 shows the proposed MSBT switch architecture of a 2 $\times 2$ optical switch where $\mathrm{I}_{1}, \mathrm{I}_{2}$ and $\mathrm{O}_{1}, \mathrm{O}_{2}$ are the input and output links, respectively. A $2 \times 2$ optical switch is used as an example for convenience of illustration. The number of switch inputs and outputs in practical applications such as wavelength division multiplexed (WDM) networks can be up to hundreds. In Fig. $1, K$ duplicates of an optical signal from an input link $\mathrm{I}_{i}$ are made by using the $1 \times K$ optical splitter. Each of the duplicated signals is delayed by $\mathrm{D}_{k}, k=1, \ldots, K$ with fiber delay lines (FDLs) and is sent to the inputs $\mathrm{I}_{i, k}, k=1, \ldots, K$ of the $2 K \times 2 K$ switching fabric. We assume that the switching

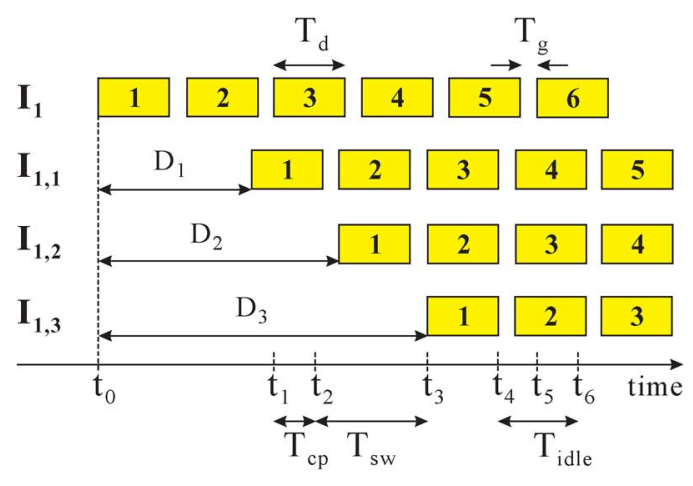

Fig. 2. Timing diagram for the packets at input link $I_{1}$ of the proposed MSBT optical switch in Fig. 1 with $K=3$, where $T_{\mathrm{d}}$ is a packet transmission time, $T_{\mathrm{g}}$ is the required guard time for preventing crosstalk between packets, and $T_{\mathrm{sw}}$ is the required reconfiguration time for switching fabric.

fabric is a commercially available internally nonblocking optical switch. The optical splitter and the FDLs form a simple optical packet serial-to-parallel transmission converter such that packets from the input link $\mathrm{I}_{i}$ will appear sequentially on the inputs $\mathrm{I}_{i, 1}$ to $\mathrm{I}_{i, K}$ of the switching fabric. A new set of input packets is therefore presented to the switching fabric every $K$ time slots. The idea is to reconfigure the switching fabric to transfer packets during another round of packet serial-to-parallel transmission conversion so that more time is available for the reconfiguration. As we will discuss in later paragraphs, $K$ can be large if the reconfiguration time $T_{\mathrm{sw}}$ of the switching fabric is larger than the transmission time $T_{\mathrm{d}}$ of a packet. Since the output signal quality of the optical splitter degrades rapidly with $K$, a fast $1 \times K$ optical switch should be used to replace the optical splitter if $K$ is large. For ease of illustration, however, the packet serial-to-parallel transmission conversion shown in Fig. 1 is assumed in this paper.

Fig. 2 shows the timing diagram for the packet transfer at the input link $\mathrm{I}_{1}$ of the proposed MSBT optical switch with $K=3$, where $T_{\mathrm{d}}$ is a packet transmission time, $T_{\mathrm{g}}$ is the required guard time for preventing crosstalk between packets, $T_{\mathrm{cp}}$ is the required time for looking up the output of an incoming packet, and $T_{\mathrm{sw}}$ is the required reconfiguration time for the switching fabric. A slot time $T_{\text {slot }}$ is equal to $T_{\mathrm{d}}+T_{\mathrm{g}}$. We assume that the output lookup operation only requires the information of the packet address and is independent of the switching fabric's status. The switching fabric can transfer packets between its inputs and outputs even if the switch is looking up the outputs for newly arrived packets. However, no packet transfer at the switching fabric is possible during the switch reconfiguration duration $T_{\mathrm{sw}}$. Hence, the switch can transfer $2 K$ packets per time period of $T_{\mathrm{sw}}+T_{\mathrm{d}}$ if we arrange the packet transfer and output lookup operations properly. To simplify the illustration in Fig. 2, we also assume that the switch immediately detects the packet address when a packet arrives at input link $\mathrm{I}_{1}$.

In Fig. 2 , packet 1 arrives at the input link $I_{1}$ at time $t_{0}$ when the system is idle. Owing to the finite switch reconfiguration time $\left(T_{\mathrm{cp}}+T_{\mathrm{sw}}=1.7\right.$ time slots $\left(T_{\text {slot }}\right)$ in Fig. 2$)$, the switching fabric is not able to directly transfer packets from $\mathrm{I}_{1}$. As shown in Fig. 2, the incoming packets are delayed by $\mathrm{D}_{1}, \mathrm{D}_{2}$, and $\mathrm{D}_{3}$ when they are sent to the inputs $\mathrm{I}_{1,1}, \mathrm{I}_{1,2}$, and 
$\mathrm{I}_{1,3}$ of the switching fabric, respectively. After reading the address of packet 3 at time $t_{1}$, the MSBT switch starts to configure the switching fabric to prepare for packet transfer at time $t_{2}=t_{0}+\mathrm{D}_{3}-T_{\mathrm{sw}}$. The packets 1,2 , and 3 are finally transferred to the switch outputs in time duration $t_{3}-t_{4}$ after the completion of the switching fabric internal path setup. From Fig. 2, one observes that the switch can also start the switching fabric internal path configuration at time $\left(t_{0}+\mathrm{D}_{2}-T_{\mathrm{sw}}\right)$ or $\left(t_{0}+\mathrm{D}_{1}-T_{\mathrm{sw}}\right)$ instead with one or two empty output slots in the initial round of packet transfer. The average added packet delay, however, remains unchanged. In Fig. 2, the switch reads packet 6 for the next round of the packet transfer process at time $t_{5}$ and starts the switching fabric reconfiguration at time $t_{6}$. The switching fabric is therefore idle for a period of $T_{\text {idle }}$ between the two reconfigurations. $T_{\text {idle }}$ is smaller than a slot time $T_{\text {slot }}$ and should be minimized for transmission bandwidth efficiency. We will discuss it in more detail when we derive the equation for the minimum value of $K$.

In Fig. 1, a $K \times 1$ optical multiplexer (MUX) and the associated $K$ sets of FDLs form an optical packet parallel-to-serial transmission converter. Packets on the outputs $\mathrm{O}_{i, 1}$ to $\mathrm{O}_{i, K}$ of the switching fabric are individually delayed and sent to the $K \times 1$ MUX to combine into the optical signal on output link $\mathrm{O}_{i}$. As the delay added to each packet should be a constant, the delays of the FDLs on the outputs $\mathrm{O}_{i, 1}$ to $\mathrm{O}_{i, K}$ are the complement of that on $\mathrm{I}_{i, 1}$ to $\mathrm{I}_{i, K}$, i.e., $\mathrm{D}_{k}+\overline{\mathrm{D}}_{k}$ is a constant. Also, a $K \times 1$ optical coupler can be used instead of the $K \times 1$ MUX in Fig. 1 if we assume that the switching fabric turns off its outputs during the reconfiguration. Otherwise, a fast $K \times 1$ optical switch is required for implementing the MUX.

For the proposed MSBT switch to operate as shown in Fig. 2, we should appropriately choose the values of $K$ and $\mathrm{D}_{i}$, $i=1, \ldots, K$. Since the switching fabric can transfer the input packets only after it completes the reconfiguration process, all packets must be delayed by at least $T_{\mathrm{cp}}+T_{\mathrm{sw}}$. In principle, any value larger than $T_{\mathrm{cp}}+T_{\mathrm{sw}}$ can be used for $\mathrm{D}_{1}$, e.g., $\mathrm{D}_{1}$ is set to 1.1 times $T_{\mathrm{cp}}+T_{\mathrm{sw}}$ in Fig. 2. To minimize the added packet delay, however, we should choose

$$
\mathrm{D}_{1}=T_{\mathrm{cp}}+T_{\mathrm{sw}} .
$$

Owing to the requirement of packet serial-to-parallel transmission conversion and minimizing the added packet delay, the delay difference between two adjacent FDLs should be equal to a slot time such that

$$
\mathrm{D}_{k}=\mathrm{D}_{k-1}+T_{\text {slot }}, \quad 2 \leq k \leq K .
$$

Equation (2) and Fig. 1 assume that the output lookup time $T_{\mathrm{cp}}$ of a packet is smaller than a slot time $T_{\text {slot }}$, or multiple processors are used such that outputs of different packets are looked up in parallel. Otherwise, the difference between $\mathrm{D}_{k}$ and $\mathrm{D}_{k-1}$ may be larger than a slot time $T_{\text {slot }}$.

To compute the minimum value of $K$, we further assume that the MSBT switch can read input packets to prepare the next round packet transfers independently of the current status of the switching fabric. The MSBT switch can also schedule multiple reconfigurations for the switching fabric. Consequently, the value of $T_{\mathrm{cp}}$ will have no effect on the minimum value of $K$ and the channel utilization. Since the switch transfers $K$ packets from an input link $\mathrm{I}_{i}$ after each switching fabric reconfiguration, the time between two switching fabric reconfigurations is therefore $K T_{\text {slot. }}$. All transmissions between the inputs and outputs of the switching fabric must be completed before the next switching fabric reconfiguration. Since $T_{\text {slot }}=T_{\mathrm{d}}+T_{\mathrm{g}}$, the maximum value of the switching fabric reconfiguration time $\left(T_{\mathrm{sw}}\right)_{\max }$ becomes $(K-1) T_{\mathrm{d}}+K T_{\mathrm{g}}$ if the $T_{\text {idle }}$ in Fig. 2 is zero. Hence, the required minimum value of $K$ can be written as

$$
K_{\min }=\left\lceil\left(T_{\mathrm{sw}}+T_{\mathrm{d}}\right) /\left(T_{\mathrm{g}}+T_{\mathrm{d}}\right)\right\rceil
$$

where $\lceil x\rceil$ is the smallest integer larger than $x$. In Fig. 2, $T_{\mathrm{sw}}$ is 1.7 time slots and the required $K_{\min }$ is therefore equal to 3. From (3), $K_{\min }$ is equal to one only if $T_{\mathrm{sw}} \leq T_{\mathrm{g}}$, i.e., the switching fabric is fast enough. Otherwise, we have to extend $T_{\mathrm{g}}$ to prevent collisions between packets as in the traditional approaches.

As the transmission rate of optical fiber grows, the reconfiguration time of large optical switches will likely be comparable to the packet transmission time in the near future. As shown in Section V, the proposed optical switch architecture will provide an excellent way to increase bandwidth efficiency. High bandwidth utilization can be achieved at the expense of added delay $\mathrm{D}_{K}$ per intermediate node. The added delay will become trivial if a packet transmission time is much smaller than the end-to-end propagation delay. However, the requirement of an $N K \times N K$ switching fabric inside an $N \times N$ switch will become a problem if $N$ is large. Traditionally, one can break a large switch into smaller switches arranged in a multistage architecture to save the hardware cost. For example, a three-stage Clos switch architecture with first and last stages of $N$ sets of $K \times K$ switches, and a second stage of $K$ sets of $N \times N$ switches can replace the $N K \times N K$ switching fabric without any difference in blocking performance [12]. In the MSBT switch application, we may only use a two-stage switch architecture instead to further reduce the required hardware. However, for optical signal quality considerations, it may be better to keep the switching fabric to one stage [10].

\section{Multifabric Sequential Transfer Switch}

Another viable solution is to replace the $N K \times N K$ switching fabric with $K$ sets of $N \times N$ switching fabrics in parallel if we transfer a packet only to its original time slot at the output link. Fig. 3 is a proposed multifabric sequential transfer (MFST) switch architecture for a $2 \times 2$ optical switch. Again, in practical applications, the MFST switch architecture will be an $N \times N$ optical switch where $N$ can be hundreds or more. With $K$ sets of $2 \times 2$ switching fabrics being connected in parallel as shown in Fig. 3, each packet can only be transferred to its original time slot at the output link. Similar to MSBT, $K$ duplicates of an optical signal from an input link $\mathrm{I}_{i}$ are made by using the $1 \times K$ optical splitter in Fig. 3, and each of them is sent to the input 


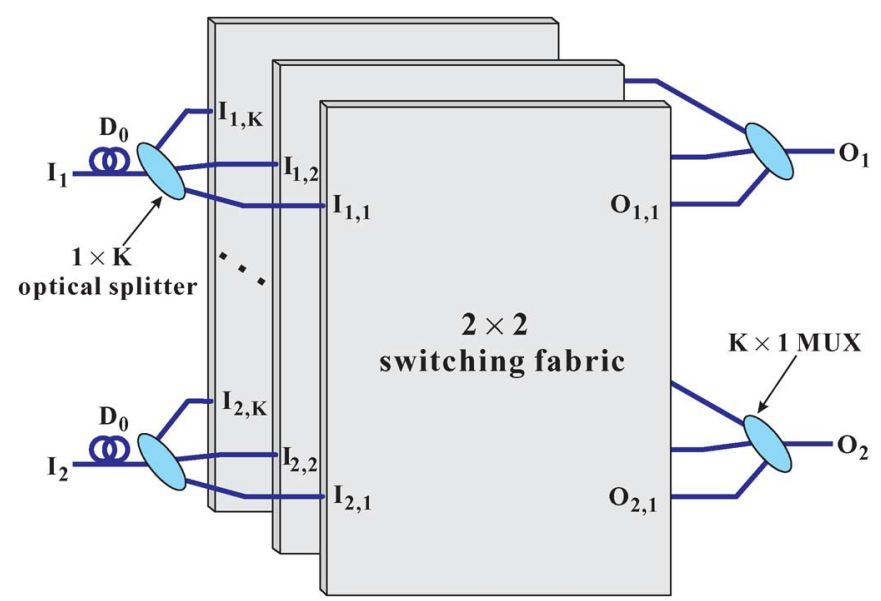

Fig. 3. Proposed multifabric sequential transfer (MFST) switch architecture for a $2 \times 2$ optical switch. Multiple smaller switching fabrics are used instead of a single large switching fabric.

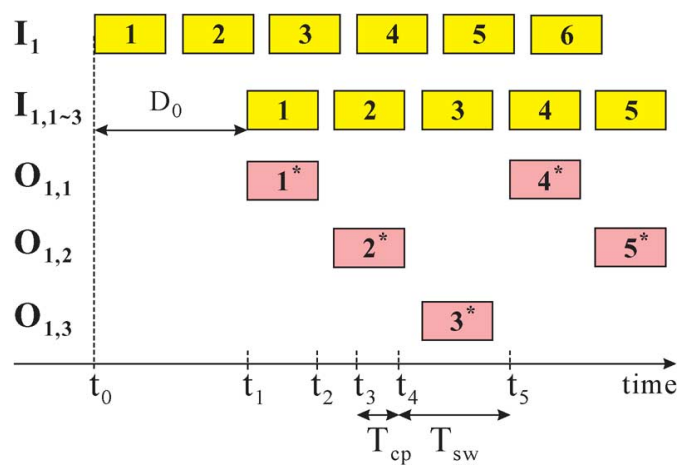

Fig. 4. Time diagram for the packets at input link $I_{1}$, at inputs $I_{1,1}$ to $I_{1,3}$, and outputs $\mathrm{O}_{1,1}$ to $\mathrm{O}_{1,3}$ of the switching fabrics of the proposed MFST $2 \times 2$ switch in Fig. 3 with $K=3$.

$\mathrm{I}_{i, k}$ of the switching fabrics, where $i=1,2$, and $k=1, \ldots, K$. However, there is no FDLs between the optical splitter and the inputs of the switching fabrics. The optical signal is first delayed $\mathrm{D}_{0}$ with the FDLs before entering the optical splitter. No FDL is required between a switching fabric output $\mathrm{O}_{i, k}$ and the input of an optical multiplexer (MUX). As in an MSBT switch, a $K \times 1$ optical coupler can be used for the MUX if each switching fabric can disable its outputs during reconfiguration.

Unlike what is shown in Fig. 1, the optical splitter in Fig. 3 and its associated FDLs do not form a packet serial-to-parallel transmission converter. Therefore, no packet serial-to-parallel conversion time is additionally available for the switching fabric reconfiguration. Since the $2 K \times 2 K$ switching fabric is decomposed into $K$ sets of $2 \times 2$ ones, the transfer of packets in different time slots will not be done in a batch. While one switching fabric is in reconfiguration, the other $K-1$ ones can freely transfer packets. Hence, the time of the packet transmissions at the other $K-1$ switching fabrics becomes available for switching fabric reconfiguration if we do the scheduling properly.

Fig. 4 shows the time diagram for the packets at input link $I_{1}$, at inputs $\mathrm{I}_{1,1}$ to $\mathrm{I}_{1,3}$, and outputs $\mathrm{O}_{1,1}$ to $\mathrm{O}_{1,3}$ of the switching fabrics of the proposed multifabric sequential transfer (MFST) 2 $\times 2$ switch with $K=3$. In Fig. 4, we assume that the total time required for packet output lookup and switching fabric reconfiguration is also equal to 1.7 time slots, i.e., $T_{\mathrm{cp}}+T_{\mathrm{sw}}=1.7 T_{\mathrm{slot}}$. We also assume that all switching fabrics detect the packet addresses at the input link $\mathrm{I}_{1}$. However, the switching fabrics are scheduled to operate in sequence such that switch fabric $k$ only starts its packet output lookup and reconfiguration at time slots $3 Z+k$, where $Z$ is a nonnegative integer and $k=1, \ldots, 3$. For example, switching fabric 1 only takes care of the packets in time slots $1,4,7, \ldots$ as shown in the Fig. 4. We assume that the packet transfer delay from the inputs to the outputs of a switching fabric is negligible. In Fig. 4, a packet $1^{*}$ (it may come from inputs $\mathrm{I}_{1,1}$ or $\mathrm{I}_{2,1}$ ) is sent to output $\mathrm{O}_{1,1}$ during time period $t_{1}$ to $t_{2}$. The switching fabric 1 then waits until time $t_{3}$ and takes $T_{\mathrm{cp}}+T_{\mathrm{sw}}$ time for the packet output lookup and internal path reconfiguration to transfer packet $4^{*}$ to output $\mathrm{O}_{1,1}$ at time $t_{5}$. During the time period $t_{2}-t_{5}$, switching fabrics 2 and 3 process the input packets 2 and 3 and transfer packets $2^{*}$ and $3^{*}$ to outputs $\mathrm{O}_{1,2}$ and $\mathrm{O}_{1,3}$ in sequence. Similarly, switching fabrics 3 and 1 will transfer packets $3^{*}$ and $4^{*}$ to outputs $\mathrm{O}_{1,3}$ and $\mathrm{O}_{1,1}$ during the reconfiguration of switching fabric 2. As the switching fabrics shift their operations in sequence, the proposed MFST switch in Fig. 3 can transfer packets between its inputs and outputs without any interruption.

In Fig. 4 , the delay $D_{0}$ can be set to any value larger than $T_{\mathrm{cp}}+T_{\mathrm{sw}}$ similar to that of $\mathrm{D}_{1}$ in Fig. 2 . The required number of switching fabrics can also be computed from (3). Apart from replacing the $N K \times N K$ switching fabric with $K$ smaller $N \times N$ ones, one advantage of the MFST switch architecture shown in Fig. 3 is that $D_{0}$ is also the total per node added delay while that of the MSBT in Fig. 1 is that $\mathrm{D}_{K}=\mathrm{D}_{1}+(K-1) T_{\text {slot }}$ from (2). With the assumption $\mathrm{D}_{0}=\mathrm{D}_{1}$, the reduction of the additional delay is $(K-1) T_{\text {slot }}$ per intermediate node, where $K \geq 2$. This will be useful if the end-to-end propagation delay is not much larger than $T_{\text {slot }}$ and the application is delay sensitive. However, $\mathrm{D}_{0}$ must be larger than $T_{\mathrm{cp}}+T_{\mathrm{sw}}$ which can cause the per node added delay of the MFST switch to be larger than $T_{\text {slot }}$. To further reduce the added delay, we need to decouple the packet address information from the packets so that a node/ switch can have prior information to configure the switching fabrics before the arrival of the input packets.

\section{Multifabric Sequential Transfer With PILOT MESSAGE}

Fig. 5 is the proposed switch architecture for multifabric sequential transfer with pilot message (MFST/PM) of a $2 \times 2$ optical switch. It is similar to that of the MFST in Fig. 3 apart from an additional switching control processor (SWCP). The switching fabrics no longer read the packet address information from the input $\mathrm{I}_{i}$. SWCP reads the packet address information from the control channels $\mathrm{C}_{\mathrm{in}-1}$ and $\mathrm{C}_{\mathrm{in}-2}$. We assume that the control channel $\mathrm{C}_{\mathrm{in}-i}$ carries messages of packet address information $T_{\text {offset }}$ time ahead of the packets on input link $\mathrm{I}_{i}$, where $i=1,2$. We also assume that SWCP needs time $T_{\mathrm{cp}}$ to detect and complete the lookup for each packet address. Once the packet output lookup is completed, SWCP instructs the switching fabrics to reconfigure the internal paths. At the same time, SWCP sends pilot messages to control channels $\mathrm{C}_{\text {out-1 }}$ and $\mathrm{C}_{\text {out-2 }}$ to inform the subsequent nodes about the 


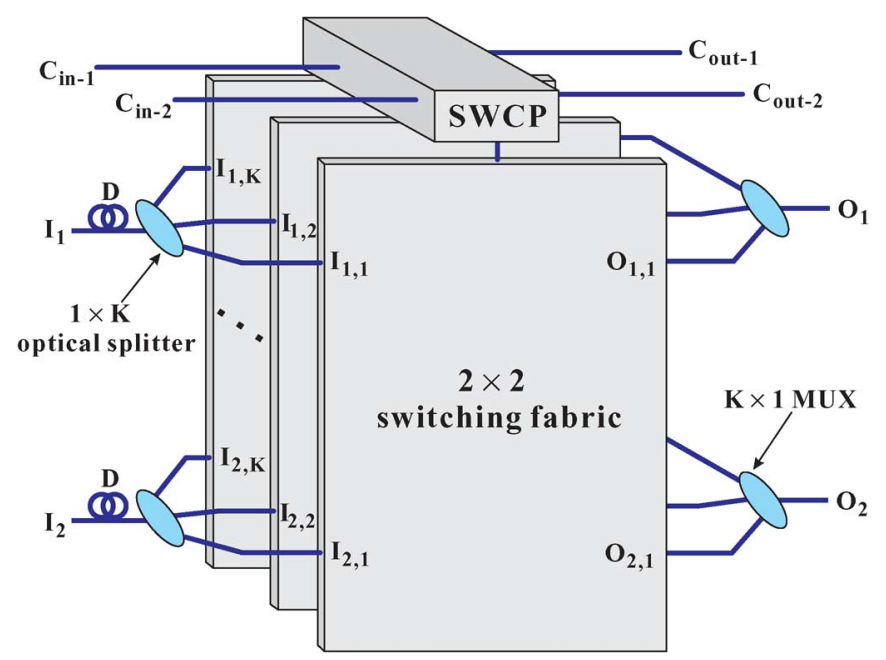

Fig. 5. Proposed multifabric sequential transfer with pilot message (MFST/PM) switch architecture for a $2 \times 2$ optical switch. It is similar to that of the MFST in Fig. 3 apart from an additional switching control processor (SWCP)

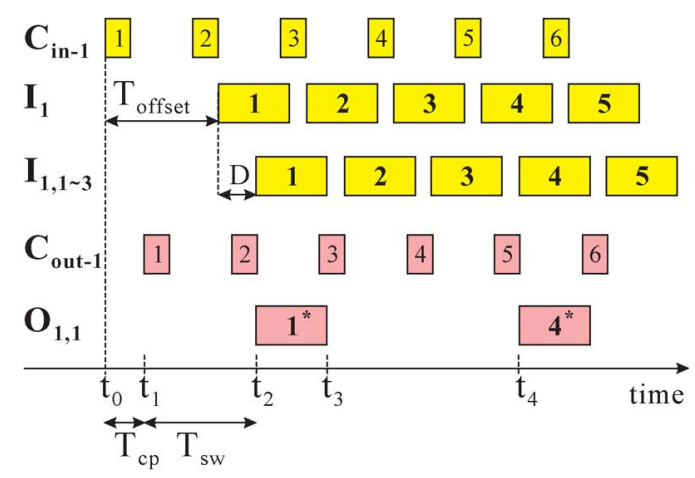

Fig. 6. Timing diagram for the packets at input link $I_{1}$, at inputs $I_{1,1}$ to $I_{1,3}$, outputs $\mathrm{O}_{1,1}$ of the switching fabric, and the pilot messages at control channels $\mathrm{C}_{\text {in-1 }}$ and $\mathrm{C}_{\text {out }-1}$ of the proposed MFST/PM $2 \times 2$ switch in Fig. 5 with $K=3$.

packets that will be sent to outputs $\mathrm{O}_{1}$ and $\mathrm{O}_{2}$ with an offset time $T_{\text {offset }}$ later. Hence, the subsequent nodes can also arrange the switching fabric settings similarly at $T_{\text {offset }}$ time before the arrival of packets. The detail of the operation is illustrated using the example in Fig. 6.

Fig. 6 is the timing diagram for the packets at input link $\mathrm{I}_{1}$, at inputs $\mathrm{I}_{1,1}$ to $\mathrm{I}_{1,3}$, outputs $\mathrm{O}_{1,1}$ of the switching fabric, and the pilot messages at control channels $\mathrm{C}_{\mathrm{in}-1}$ and $\mathrm{C}_{\text {out }-1}$ of the proposed MFST/PM $2 \times 2$ switch with $K=3$. We assume the same $T_{\mathrm{cp}}$ and $T_{\mathrm{sw}}$ as that of Figs. 2 and 4. In Fig. 6, the pilot message 1 arrives at time $t_{0}$. SWCP takes time $T_{\mathrm{cp}}$ to look up the output and therefore the switching fabric starts the internal path reconfiguration at time $t_{1}$. To compensate for the pilot message processing time at SWCP, the FDLs at each input link $\mathrm{I}_{i}$ has delay value of $D \geq T_{\mathrm{cp}}$. Therefore, at time $\mathrm{t}_{1}$, SWCP has the complete information of the whole switch in the next $T_{\text {offset }}$ time, e.g., packet $1^{*}$ will be sent to output $\mathrm{O}_{1,1}$ at time $t_{2}$. If packet $1^{*}$ is a new packet, we assume that it is delayed at least $T_{\mathrm{cp}}+T_{\mathrm{sw}}$ time before it is allowed to enter the switch, i.e., packet $1^{*}$ has to arrive at the switch before time $t_{0}$ such that SWCP will include it in the output assignment at

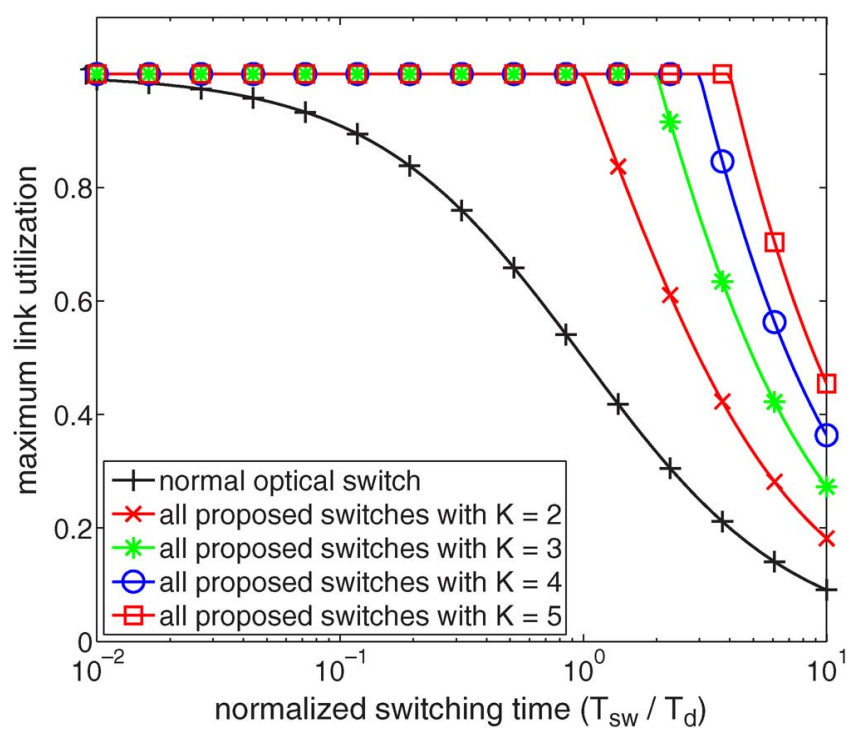

Fig. 7. Maximum link utilization of switch outputs for the proposed MSBT, MFST, and MFST/PM switch architectures. The curves with crosses, asterisks, circles, and squares are the maximum output link utilization of the proposed switches with $K=2,3,4$, and 5 , respectively. The curve with pluses is that of the normal optical switch, i.e., the proposed switches with $K=1$.

$t_{1}$. Hence, SWCP sends out the pilot message 1 to the control channel $\mathrm{C}_{\text {out }-1}$ at time $\mathrm{t}_{1}$ without waiting for the completion of the switching fabric reconfiguration. With the assumption of using the same routing paths of their associated packets, pilot messages will arrive at the subsequent nodes $T_{\text {offset }}$ time ahead, and the subsequent nodes can preconfigure their switching fabrics accordingly.

The minimum added delay of the MFST/PM switch in Fig. 5 will be $H \times T_{\mathrm{cp}}+T_{\mathrm{sw}}$ for an $H$ hop path if we assume that $\mathrm{D}=T_{\mathrm{cp}}, T_{\text {offset }}=T_{\mathrm{sw}}$, and a packet is filtered out before the input of the destination switch. The minimum delay includes the $T_{\mathrm{cp}}+T_{\mathrm{sw}}$ initial delay at the source and the $T_{\mathrm{cp}}$ delay at each intermediate node. The intermediate node switching fabric reconfiguration time, however, becomes irrelevant to the end-to-end delay because it overlaps with the delay time at the source and the node-to-node traveling time of the packets, just as in OBS [6], [7]. Since the $H$ hop path minimum added delays of the MSBT switch in Fig. 1 and MFST switch in Fig. 3 are $H \times$ $\left(T_{\mathrm{cp}}+T_{\mathrm{sw}}+(K-1) T_{\mathrm{slot}}\right)$ and $H \times\left(T_{\mathrm{cp}}+T_{\mathrm{sw}}\right)$, respectively, the delay savings with the MFST/PM switch in Fig. 5 will be significant if the reconfiguration time of the switching fabric is large.

\section{Performance Evaluation}

\section{A. Link Utilization}

Fig. 7 shows the maximum (achievable) link utilization of the switch outputs for the proposed MSBT, MFST, and MFST/PM switch architectures provided that the guard time for preventing the crosstalk between packets is not required. Recall that the utilizations of the different proposed switches are the same. In Fig. 7, the curves with crosses, asterisks, circles, and squares are the maximum output link utilizations of the proposed switches with $K=2,3,4$, and 5, respectively, 
where, $K$ is the packet serial-to-parallel conversion ratio of the MSBT switch and the number of switching fabrics of the MFST and MFST/PM switches. The curve with pluses is that of the normal optical switch, i.e., it is equal to the proposed switches with $K=1$. The horizontal axis is the switch reconfiguration time normalized by the packet transmission time, i.e., $T_{\mathrm{sw}} / T_{\mathrm{d}}$. The maximum link utilization in the vertical axis is calculated using $T_{\mathrm{d}} /\left(T_{\mathrm{d}}+T_{\mathrm{g}}\right)$ where the interpacket guard time $T_{\mathrm{g}}$ is set to the minimum value to get the required $K$ in (3) with the given $T_{\mathrm{sw}}$ and $T_{\mathrm{d}}$. From (3), we have

$$
T_{\mathrm{g}}= \begin{cases}\left(\left(T_{\mathrm{sw}} / T_{\mathrm{d}}\right)-K+1\right) / T_{\mathrm{d}} & \text { if }\left(T_{\mathrm{sw}} / T_{\mathrm{d}}\right)>K-1 \\ 0 & \text { otherwise. }\end{cases}
$$

We assume that the delays $D_{1}$ to $D_{K}$ of the FDLs in Fig. 1 are changed accordingly. In Fig. 7, we vary the normalized switching time $T_{\mathrm{sw}} / T_{\mathrm{d}}$ between 0.01 and 10 to show the effect of switch reconfiguration time variations. For a normal optical switch, the switching overhead in general should only be a small fraction of the packet length, e.g., $T_{\mathrm{sw}} / T_{\mathrm{d}}<0.1$. Otherwise, the link utilization will drop rapidly as shown in Fig. 7. The maximum link utilization for normal optical switch drops to 0.5 when $T_{\mathrm{sw}} / T_{\mathrm{d}}=1$. In contrast, all curves for the proposed switches remain at unity until $T_{\mathrm{sw}} / T_{\mathrm{d}}$ is larger than $K-1$. With the proposed switches, the tolerable switch reconfiguration time $T_{\mathrm{sw}}$ increases from a fraction of a packet transmission time to several packet transmission times. While packets typically have lengths of kilobytes, we can use the switching fabrics with reconfiguration time a thousand times of that normally required for optical packet switching, e.g., the maximum length of an Ethernet packet is 1522 bytes [13].

\section{B. Added Packet Delay}

Fig. 8 shows the average added end-to-end delay to the packets in an $8 \times 8$ Manhattan Street Network (MSN) [14] with the proposed switch architectures for MSBT, MFST, and MFST/PM. In the $8 \times 8 \mathrm{MSN}$, the average path length is 5.016 hops. We set the packet transmission time $T_{\mathrm{d}}$ to one time unit. As in Fig. 7, we increase the switching fabric reconfiguration time $T_{\mathrm{sw}}$ from 0.01 to 10 of the $T_{\mathrm{d}}$. However, both the packet output lookup time $T_{\mathrm{cp}}$ and the interpacket guard time $T_{\mathrm{g}}$ are fixed at $0.1 T_{\mathrm{d}}$. Hence, a slot time $T_{\text {slot }}$ is equal to 1.1 unit time. The added delays of MSBT, MFST and MFST/PM switches are therefore $5.016 \times\left[0.1+T_{\mathrm{sw}}+1.1 \times(K-1)\right]$, $5.016 \times\left(0.1+T_{\mathrm{sw}}\right)$ and $5.016 \times 0.1+T_{\mathrm{sw}}$, respectively. As the switching fabric reconfiguration time $T_{\mathrm{sw}}$ increases, $K$ is determined by (3). Hence, the value of $K$ increases from 1 to 10 when $T_{\mathrm{sw}}$ changes from 0.01 to 10 .

In Fig. 8, the curves with dots, triangles and diamonds are the average end-to-end added delay of the $8 \times 8$ MSN network with MSBT, MFST, and MFST/PM switches, respectively. The difference between the added delays of the three proposed switches is small when $T_{\mathrm{sw}}$ is below 0.1 , where $K$ is equal to one. The added delay of the MSBT switch is equal to that of the MFST switch, and is only around $4 T_{\mathrm{sw}}$ larger than that of the MFST/PM switch. When $T_{\mathrm{sw}}$ is larger than 0.1, the added delay of MSBT increases rapidly because of the increase of

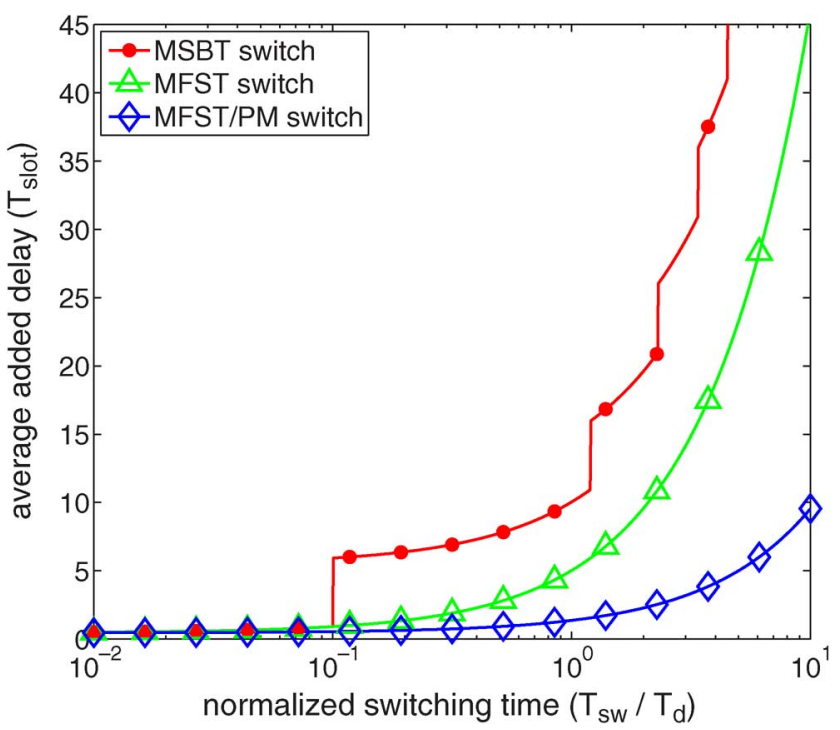

Fig. 8. Average added end-to-end delay to the packets in an $8 \times 8$ Manhattan Street Network (MSN) [14] with the proposed switches that we have presented in Sections II, III, and IV. The curves with dots, triangles and diamonds are the average end-to-end added delay of the $8 \times 8 \mathrm{MSN}$ network with MSBT, MFST, and MFST/PM switches, respectively.

$K$. At $T_{\mathrm{sw}}=10$, the added delays of the MSBT, MFST, and MFST/PM switches are 91.2 (not shown in Fig. 8), 46.1, and 9.5 time slots $\left(T_{\text {slot }}\right)$, respectively. As shown in the Fig. 8, the MFST/PM switch can significantly reduce the added delay if the switch reconfiguration time $T_{\mathrm{sw}}$ is a multiple of the packet transmission time $T_{\mathrm{d}}$. Since the MFST/PM switch requires additional signaling of pilot messages in the network, the MFST approach in general should be used unless $T_{\mathrm{sw}}$ is really large.

\section{Packet Discarding Performance}

All the proposed switch architectures for MSBT, MFST, and MFST/PM are internally nonblocking switches. However, the MSBT switch can further reduce output blocking by allowing the packets to shift from their original time slots at the output of the switch. This function can be implemented without time slot interchanger by transferring a packet from input $\mathrm{I}_{i, j}$ (a packet from the slot $j$ of the $K$ packet batch on input link $i$ ) of the switching fabric to any output $\mathrm{O}_{m, n}$ for $1 \leq n \leq K$, not just to output $\mathrm{O}_{m, j}$ only, where $m$ is the preferred output of the packet. Thus, $K$ time slots are available to the packet instead of only the original time slot. To demonstrate the effect of $K$ on reducing the packet loss probability of the MSBT switches, we define $\mathrm{p}_{i}(L)$ as the probability of having $L$ other contending packets for an output link $\mathrm{O}_{i}$ when a packet is at input $\mathrm{I}_{i, k}$, and the MSBT switch is starting to look up the outputs of the input packets, i.e., at time $\mathrm{t}_{1}$ in Fig. 2 , where $1 \leq i \leq N, 1 \leq k \leq K$, $0 \leq L \leq(N K-1)$, and $N$ is the number of input/output links. We assume that the arriving packets choose the output links at random. Hence, we have

$$
\mathrm{p}_{i}(L)=\sum_{M=L}^{N K-1} \mathrm{q}(M)\left(\begin{array}{c}
M \\
L
\end{array}\right)\left(\frac{1}{N}\right)^{L}\left(\frac{N-1}{N}\right)^{M-L}
$$




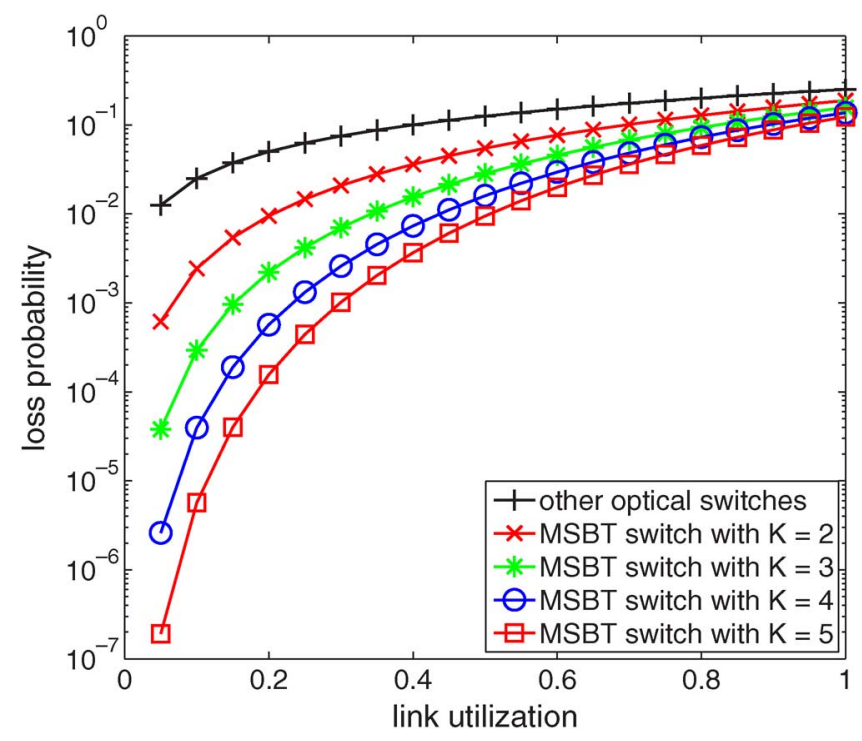

Fig. 9. Packet loss probability of the proposed MSBT optical switch with two input/output links ( $N=2)$ and different values of $K$. Equations (4)-(6) are used for the loss probability calculation. We assume that packets arrive randomly at input links and choose the output links at random. Other notations are similar to that of Fig. 7.

where $\mathrm{q}(M)$ is the probability of $M$ other packets arriving at other inputs of the switching fabric. $\mathrm{p}_{i}(L)$ is the binomial distribution with parameters $M, L$, and $1 / N$ when $M$ is fixed. Assuming that the packets arrive uniformly at all input links, $M$ is also a binomial random variable. We have

$$
\mathrm{q}(M)=\left(\begin{array}{c}
N K-1 \\
M
\end{array}\right) \rho^{M}(1-\rho)^{N K-M-1}
$$

where $\rho$ is the utilization of the input links. Since the packets choose output links at random, the average packet loss probability is equal to that of the packet being discarded at any output link $\mathrm{O}_{i}$. Assuming that each packet has the same priority, the average packet loss probability $\mathrm{B}$ can be written as

$$
B=\sum_{L=K}^{N K-1} \mathrm{p}_{i}(L) \frac{L-K+1}{L+1} .
$$

Fig. 9 shows the packet loss probability of the MSBT switch with two input and two output links $(N=2)$ for different values of $K$. As in Fig. 7, the curves with crosses, asterisks, circles, and squares represent the loss probabilities of the proposed MSBT switch with $K=2,3,4$, and 5 , respectively. The curve with pluses is the packet loss probability of the normal optical switch. It is also the loss probability of the MSBT switch with $K=1$ and that of the MFST and MFST/PM switches. From Fig. 9, the packet loss probability of the MSBT switch decreases when $K$ increases, especially when the system is lightly loaded. Thus, the proposed MSBT switch architecture can greatly improve packet loss performance in addition to easing the constraint on the switch response time. However, the reduction in loss probability (compared with the loss probability when $K=1$ ) decreases when the link utilization increases.

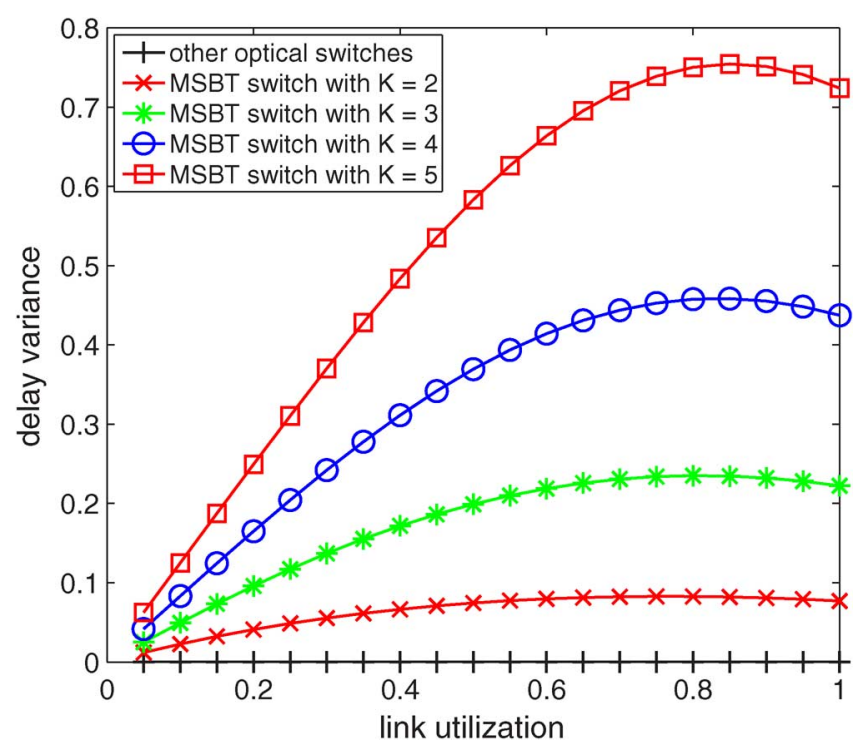

Fig. 10. Packet delay variance of the proposed MSBT optical switch with $N=$ 2 and different values of $K$. We assume that the packets will choose any available output of switching fabric at random if the default output is unavailable. Other assumptions and notations are similar to that of Fig 7.

\section{Delay Variance}

As we have discussed in Section II, the FDLs in the outputs and inputs of the MSBT switch are complementary to each other, i.e., $\mathrm{D}_{k}+\overline{\mathrm{D}}_{k}$ is a constant. Transferring a packet to a time slot other than its original time slot at the output link will cause a delay fluctuation of up to $\pm(K-1)$ time slots. Using (6), we define $\mathrm{B}_{N}(K)$ as the packet loss probability on an $N \times N$ MSBT switch with $K$ serial-to-parallel packet conversion ratio. Hence, $\mathrm{B}_{N}(1)$ is also the loss probability of the MSBT switch if only the original time slot can be used for the packet transfer, and $\mathrm{P}_{n}=$ $\left(\mathrm{B}_{N}(1)-B_{N}(K)\right) /\left(1-\mathrm{B}_{N}(K)\right)$ is the probability of a packet not transferred to its own time slot. We assume that a packet is assigned to any available one of the $K$ time slots at random if the default time slot is unavailable. Let $x$ be the delay fluctuation of a transferred packet. If a packet arrives at the $k$ th of the $K$ time slots, $x$ will have probability $\left(1-\mathrm{P}_{n}\right)$ to be 0 and $\mathrm{P}_{n} /(K-1)$ to be one of the $K-1$ values of $(-(k-1), \ldots,-1,1, \ldots, K-k)$, where $1 \leq k \leq K$. Since packets arrive randomly at each time slot and switch input, the probability distribution of $x$ is $\mathrm{P}(x=$ $z)=\mathrm{P}_{n} \times(K-|z|) / K /(K-1)$, where $|z|$ is the absolute value of $z$, and $-(K-1) \leq z \leq(K-1)$. We have the expectation $\mathrm{E}(x)=0$ and the variance $\operatorname{Var}(x)=\mathrm{E}\left(x^{2}\right)-[\mathrm{E}(x)]^{2}$ to be the delay variance of a transferred packet in MSBT switches.

Fig. 10 shows the packet delay variance of the MSBT switch with $N=2$ and different values of $K$. In Fig. 10, there is no packet delay variance for other proposed switches, i.e., the curve with the pluses. For the MSBT switch, the delay variance increases with $K$ and also increases with the link utilization in the range $0-0.8$. Figs. 9 and 10 show that the packet loss probability of the MSBT switches may be reduced at the expense of additional delay and delay variance to the packets. We can maintain the packet sequence integrity by optimizing the switch output port to packet assignment, but the packet delay variance cannot be avoided unless additional optical hardware such as 
optical time slot interchangers are used. Since packets can accumulate significant delay variance along the path, large buffers are required at the end nodes to smooth out the jitters if real time traffic is carried in the networks.

\section{IMPLEMENTATION CONSIDERATIONS}

In this paper, we propose a way to increase the throughput of optical networks by using batch transfer of packets or multiple switching fabrics in parallel. We have proposed the MSBT, MFST, and MFST/PM switch architectures to overcome the stringent demand on the optical switching speed and the relative duration of the guard time between packets. Many issues must be considered for the realization of the proposed switches, including the power consumption and dissipation, scalability, length of the optical delay lines required, complexity of the scheduler, and cascadability. In the following, we will discuss some of the implementation issues.

Power consumption is an important issue in the design of future routers because power consumption by data centers nowadays can rival that of a small town. For the proposed switches, power consumption in the worst case is proportional to the square of the number of ports $N$ if technologies such as the semiconductor optical amplifier (SOA) crossbar structure are used to build the switching fabrics [4]. Since MBST switches use a single $K N \times K N$ switching fabric, the worst case power consumption will be proportional to $K^{2} N^{2}$ times the power consumed by individual switching element. Large power consumption will also affect the scalability of multiple routers in parallel configurations. The proposed MFST and MFST/PM switches use $K$ switching fabrics in parallel and therefore the power consumption is proportional to $K$ times that of individual switching fabric. In general, the MFST and MFST/PM switches have smaller power consumption, and will have better scalability than MSBT switches.

It is well known that FDLs are large and bulky. One meter of FDLs introduces $5 \mathrm{~ns}$ of delay. A packet with transmission time $T_{\mathrm{d}}$ of $1.5 \mu \mathrm{m}$ needs at least $300 \mathrm{~m}$ of FDLs to store. In the proposed MSBT switches, delays of multiple packet time duration are required for the packet serial-to-parallel and parallel-to-serial transmission converters. Thus, kilometer long FDLs will have to be used. Hence, MSBT switches will face the same problems as other switches with optical buffers if the number of ports $N$ and the serial-to-parallel ratio $K$ are large. Since MFST and MFST/PM switches require smaller packet delay (it is independent of $T_{\mathrm{d}}$ ) and only one set of FDLs is used for an input port, these switches will use much less FDLs than MSBT switches. From this consideration, the smaller packet delay of MFST and MFST/PM switches not only can improve the system performance but also has advantages in implementation of the proposed switches.

Using a 1-to- $K$ optical power splitter ( $K$-to- 1 power combiner) to implement the packet de-multiplexer (packet multiplexer) can introduce a large insertion loss. For example, in a 1-to-64 splitter, the packets will experience more than 36 $\mathrm{dB}$ of power losses. This is another reason that we prefer to have a small $K$. As shown in Section $\mathrm{V}$-A, we can use switching fabrics with reconfiguration time $T_{\mathrm{sw}}$ up to $(K-1)$ packet transmission time $T_{\mathrm{d}}$ in our proposed switches to have nearly $100 \%$ link utilization. If $T_{\mathrm{d}}$ is in the order of microseconds, optical switches with microsecond reconfiguration time can be used for the switching fabrics. Thus, MEMS switches are in general not suitable as the switching fabrics because of their millisecond reconfiguration time. Recently, an $8 \times 8$ PLZT $\left((\mathrm{Pb}, \mathrm{La})(\mathrm{Zr}, \mathrm{Ti}) \mathrm{O}_{3}\right)$ electro-optic switch (port count extension capability up to 64 ports) with a microsecond reconfiguration time has been demonstrated [15]. A $64 \times 64$ GaAs phased array electro-optic switches can even achieve a reconfiguration time of $30 \mathrm{~ns}$ but with the drawback of polarization dependence [16]. Hence, $K=2$ or $K=4$ should be generally sufficient. For a larger but moderate value of $K$, such as $K=8$, we have proposed to use $1 \times K$ and $K \times 1$ optical switches to replace the splitters and combiners in all MSBT, MFST, and MFST/PM switches. Optical switches such as the 1 $\times 8$ PLZT optical switches with potential loss of $6 \mathrm{~dB}$ and $T_{\mathrm{sw}}$ around $10 \mathrm{~ns}$ are available [17]. If $T_{\mathrm{d}}$ is so small that $K$ has to be large, optical power splitters and combiners will have to be used for packet de-multiplexing and multiplexing but then additional signal amplification is needed to compensate for the power losses. Of course, the system complexity will increase.

The proposed switches continually process the packets and the switch schedulers have to assign each packet a suitable output within a slot time, i.e., $T_{\mathrm{cp}}<T_{\text {slot }}$. When the optical fiber transmission rate increases and $T_{\text {slot }}$ becomes small, the performance of the switch scheduler will become a limiting factor for the system throughput. Many approaches have been used to improve the scheduler performance. They include efficient lookup algorithms [18], better routing table designs [19], hardware-based lookup methods [20], and elimination of the lookup process by using self-routing schemes [21]. In this paper, we focus on the transmission bandwidth overhead caused by the switch reconfiguration time, and simply assume that the switch schedulers have the required performance.

A physical control channel is not necessary for MFST/PM switches because we can embed the pilot messages into the earlier arriving packets though it may add a delay slightly larger than the minimum value, e.g., pilot messages 3 and 4 can be carried in packets 1 and 2 of Fig. 6. Since MSFST/PM switches use the pilot messages to shorten the packet delay, it is necessary that the same type of switching fabrics (with the same reconfiguration time) is used at all nodes. In contrast, both MSBT and MFST switches do not need pilot messages and they have greater flexibility of using different types of switching fabrics at different nodes.

In this paper, we have only discussed the basic architectures of the proposed switches. Many services can be provided by the proposed switches with no or minor modifications. For example, using multichannel deflection routing to resolve packet contention has been proposed for networks with nodes of MSBT switches [22]. The analytical model for throughput delay performance of the MSBT networks has been derived. Also, multicast service can be implemented on MSBT switches if multiple delay switchable FDLs are used in the serial-to-parallel packet converter. Finally, the MSBT switches with switchable FDLs can provide other important services such as packet buffering and priority routing. 


\section{CONCLUSION}

We have studied the use of batch transfer of packets and multiple switching fabrics in parallel to relax the stringent constraint imposed by the switch reconfiguration time on optical packet switched networks. All three proposed switch architectures can provide the same bandwidth utilization improvement but with different added packet delays and implementation requirements.

In the MSBT switch architecture, we use packet serial-to-parallel transmission conversion to retrieve the future information of a batch of arriving packets and use the gained time to preconfigure the switching fabric. Although the MSBT switch can relax the requirement of switch reconfiguration time from a small fraction of the packet transmission time to multiple packet transmission times, it requires a large single switching fabric and may be difficult to implement. We therefore, also propose the multifabric sequential transfer (MFST) architecture that uses multiple smaller size switching fabrics instead. Apart from simplifying the implementation, the MFST also greatly reduces the added delay to packets compared to that of the MSBT switch. To further reduce the added packet delay, we decouple the routing information from the packets and propose the MFST with pilot message (MFST/PM) switch architecture.

Although the MSBT architecture uses a single large switching fabric and has the largest added packet delay, it can reduce the packet loss probability without extra hardware by not limiting packet transfer only to its original time slot at the output link. However, large buffers will be required at the end nodes of the network because of the increased delay variance.

\section{REFERENCES}

[1] S. Aisawa, T. Sakamoto, M. Fukui, J. Kani, M. Jinno, and K. Oguchi, "Ultra-wideband, long distance WDM demonstration of $1 \mathrm{Tbit} / \mathrm{s}(50 \times$ $20 \mathrm{Gbit} / \mathrm{s}), 600 \mathrm{~km}$ transmission using 1550 and $1580 \mathrm{~nm}$ wavelength bands," Electron. Lett., vol. 34, no. 11, pp. 1127-1129, 1998.

[2] R. Van Caenegem, "From IP over WDM to all-optical packet switching: Economical view," J. Lightw. Technol., vol. 24, no. 4, pp. 1638-1645, 2006.

[3] T. Houbavlis, "All-optical signal processing and applications within the Esprit project DO_ALL," J. Lightw. Technol., vol. 23, no. 2, pp. 781-801, 2005.

[4] G. I. Papadimitriou, C. Papazoglou, and A. S. Pomportsis, "Optical switching: Switch fabrics, techniques, and architectures," J. Lightw. Technol., vol. 21, no. 2, pp. 384-405, 2003.

[5] J. Kim, "1100 × 1100 port MEMS-based optical crossconnect with 4-dB maximum loss," IEEE Photon. Technol. Lett., vol. 5, no. 11, pp. 537-1539, 2003

[6] C. Qiao and M. Yoo, "Optical burst switching (OBS) — A new paradigm for an optical Internet," J. High Speed Netw., vol. 8, pp. 69-84, 1999.

[7] C. Y. Li, G. M. Li, P. K. A. Wai, and V. O. K. Li, "Optical burst switching with large switching overhead," IEEE J. Lightw. Technol., vol. 25, no. 2, pp. 451-462, Feb. 2007.

[8] [Online]. Available: http://www.mfaforum.org/tech/atm_specs.shtml

[9] C. Y. Li, P. K. A. Wai, X. C. Yuan, and V. O. K. Li, "Deflection routing in slotted self-routing networks with arbitrary topology," IEEE J. Sel. Area Commun., vol. 22, no. 9, pp. 1812-1822, Nov. 2004.

[10] L. Y. Lin, E. L. Goldstein, and R. W. Tkach, "On the expandability of free-space micromachined optical cross connects," J. Lightw. Technol., vol. 8, no. 4, pp. 482-489, 2000.

[11] T. Nakahara, R. Takahashi, and H. Suzuki, "Self-routing of 100-Gb/s optical packets using self serial-to-parallel conversion-based label recognition," IEEE Photon. Technol. Lett., vol. 15, no. 4, pp. 602-604, Apr. 2003
[12] J. Y. Hui, Switching and Traffic Theory for Integrated Broadband Networks. : Kluwer Academic Publishers, Boston, 1990.

[13] Frame Extensions for Virtual Bridged Local Area Network (VLAN) Tagging on 802.3 Networks, IEEE Standard 802.3ah, 2004.

[14] N. F. Maxemchuk, "Routing in Manhattan Street Network," IEEE Trans. Commun., vol. 35, no. 5, pp. 503-512, May 1987.

[15] A. Sugama, "Integrated $8 \times 8$ electro-optic high-speed switch for optical burst transport networks," presented at the Optical Fiber Communication Conference (OFC 2007), 2007, paper OWV2, unpublished.

[16] T. McDermott and T. Brewer, "Large-scale IP router using a high-speed optical switch element," J. Opt. Network., vol. 2, no. 7, pp. 229-240, 2003.

[17] M. Hayashitani, "10 ns high-speed PLZT optical content distribution system having slot-switch and GMPLS controller," IEICE Electron. Expr., vol. 5, no. 6, 2008.

[18] M. A. Ruiz-Sanchez, E. W. Biersack, and W. Dabbous, "Survey and taxonomy of IP address lookup algorithms," IEEE Netw., vol. 15, no. 2, pp. 8-23, Mar. 2001.

[19] S. Sahni and K. S. Kim, "An O(logn) dynamic router-table design," IEEE Trans. Comput., vol. 53, no. 3, pp. 351-363, Mar. 2004

[20] M. J. Akhbarizadeh and M. Nourani, "Hardware-based IP routing using partitioned lookup table," IEEE/ACM Trans. Networking, vol. 13, no. 4, pp. 769-781, Aug. 2005.

[21] X. C. Yuan, V. O. K. Li, C. Y. Li, and P. K. A. Wai, "A novel selfrouting scheme for all-optical packet switched networks with arbitrary topologies," J. Lightw. Technol., vol. 21, no. 2, pp. 329-339, 2003.

[22] C. Y. Li, P. K. A. Wai, and V. O. K. Li, "Performance model of deflection-routed multislot batch-transfer networks," in Proceedings of 51th Annual IEEE Global Communications Conference (Globecom 2008), New Orleans, LA, USA, 30 November-4 December 2008.

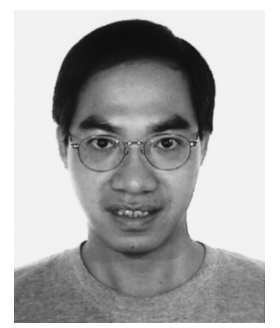

C. Y. Li (S'91-M'93) received the B.Sc. degree from the National Taiwan University, Taiwan, in 1986, and the Ph.D. degree from The Hong Kong Polytechnic University, Hong Kong, in 2000.

In 1986, he joined Taicom Ltd., Taiwan as a Transmission Engineer, and worked on the M90, M135, and M405 Optical Fiber Telecommunication System projects. In 1988, he joined ROCTEC Ltd., Hong Kong, as a Design Engineer, and worked on the computer products development. In 1993, he joined the Department of Electronic and Information Engineering, Hong Kong Polytechnic University, as a Research Assistant.

His current research interests include optical switch architectures, network performance evaluation, all-optical network routing, and network theory.

Dr. Li has authored or coauthored over 30 papers in these areas.

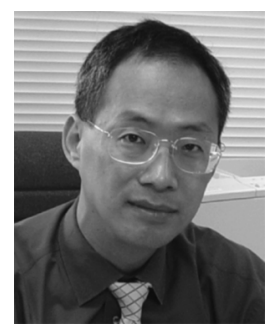

Ping kong Alexander Wai (SM'96) received the Bachelor of Science (Hons) degree from the University of Hong Kong, Hong Kong, in 1981 and the Ph.D. degree from the University of Maryland, College Park, in 1988.

After graduation, he joined Science Applications International Corporation, McLean, VA, where he worked on the Tethered Satellite System Project which is a space shuttle mission. Then he worked in the Department of Electrical Engineering, University of Maryland, on optical fiber transmission systems. In 1996, he joined the Department of Electronic and Information Engineering, The Hong Kong Polytechnic University. Currently, he is the Chair Professor in Optical Communications. He also serves as Dean of Faculty of Engineering and Associate Vice President. His research interests include optical solitons optical fiber communication systems; all-optical packet switching; fiber lasers and amplifiers; optical networks; computational photonics; network theories. He has published over 100 articles in archival journal and had served in the program committees of a number of international conferences.

Dr. Wai is the General Chair of the 14th Optoelectronics and Communications Conference held in Hong Kong, 2009. He is an Associate Editor of Optics Express and a Fellow of Optical Society of America. 


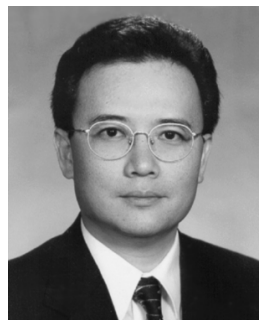

Victor O. K. Li (S'80-M'81-SM'86-F'92) received SB, SM, EE and ScD degrees in electrical engineering and computer science from Massachusetts Institute of Technology, Cambridge, MA, in 1977, 1979, 1980, and 1981, respectively. In 1981, he joined the University of Southern California (USC), Los Angeles, CA, and became Professor of Electrical Engineering and Director of the USC Communication Sciences Institute. Since September 1997 he has been with The University of Hong Kong, Hong Kong, where he is Associate Dean (Research) of Engineering, and Chair Professor of Information Engineering, Department of Electrical and Electronic Engineering. He was the Managing Director of Versitech Ltd., the technology transfer and commercial arm of the University, and on various corporate boards. His current research interests include information technology, including all-optical networks, wireless networks, and Internet technologies and applications.

Prof. Li was the Chair of the Computer Communications Technical Committee of the IEEE Communications Society during 1987-1989, and the Los Angeles Chapter of the IEEE Information Theory Group during 1983-1985. $\mathrm{He}$ co-founded the International Conference on Computer Communications and Networks (IC3N), and was the Chair of its Steering Committee during 1992-1997. He was also the Chair of various international workshops and conferences, including, most recently, IEEE INFOCOM 2004 and IEEE HPSR
2005. Prof. Li has served as an editor of IEEE Network, IEEE JSAC Wireless Communications Series, IEEE Communications Surveys and Tutorials, and Telecommunication Systems. He also guest edited special issues of IEEE JSAC, Computer Networks and ISDN Systems, and KICS/IEEE Journal of Communications and Networking. He is now serving as an editor of ACM/Springer Wireless Networks. Prof. Li has been appointed to the Hong Kong Information Infrastructure Advisory Committee by the Chief Executive of the Hong Kong Special Administrative Region (HKSAR). He was a part-time member of the Central Policy Unit of the Hong Kong Government. He was also with the Innovation and Technology Fund (Electronics) Vetting Committee, the Small Entrepreneur Research Assistance Programme Committee, and the Engineering Panel of the Research Grants Council. He was a Distinguished Lecturer at the University of California at San Diego, at the National Science Council of Taiwan, and at the California Polytechnic Institute. He has also delivered keynote speeches at many international conferences.

Prof. Li has received numerous awards, including, most recently, the PRC Ministry of Education Changjiang Chair Professorship at Tsinghua University, the UK Royal Academy of Engineering Senior Visiting Fellowship in Communications, the Outstanding Researcher Award of The University of Hong Kong, the Croucher Foundation Senior Research Fellowship, and the Order of the Bronze Bauhinia Star, Government of HKSAR, China. He is a Fellow of the Hong Kong Institution of Engineers and the IAE. 